\title{
The heliospheric modulation of electrons and positrons over a complete solar cycle
}

\author{
Aslam, O. P. M.* \\ Centre for Space Research, North-West University, 2520 Potchefstroom, South Africa \\ E-mail: aslamklr2003@gmail.com \\ Driaan Bisschoff \\ Centre for Space Research, North-West University, 2520 Potchefstroom, South Africa \\ E-mail: driaanbegmail.com
}

\section{Marius S Potgieter}

Centre for Space Research, North-West University, 2520 Potchefstroom, South Africa

E-mail: marius.potgieter@nwu.ac.za

\begin{abstract}
The solutions of a comprehensive three-dimensional drift model are compared to PAMELA and AMS-02 spectra of electrons and positrons in order to describe and understand their different modulation down to $1 \mathrm{MeV}$. This is based on newly constructed very local interstellar spectra. The focus of the study is on a full solar cycle, the period from 2006 to 2017. Comparison of observations and modeling provides insight into how the three major diffusion coefficients change during a complete solar cycle, especially during a prolonged solar minimum and the period of the polarity reversal of the magnetic field during solar maximum activity, and to what extent drift effects evolve. In this context, the electron to positron ratio is computed from $1 \mathrm{MeV}$ to $50 \mathrm{GeV}$, explaining the PAMELA and AMS-02 observed ratio in detail. Predictions are made of what may be observed during the next, imminent solar minimum.
\end{abstract}

36th International Cosmic Ray Conference -ICRC2019-

July 24th - August 1st, 2019

Madison, WI, U.S.A.

${ }^{*}$ Speaker. 


\section{Introduction}

The turbulent solar wind, and the heliospheric magnetic field (HMF) embedded in the solar wind modulates cosmic rays (CRs) in the heliosphere. In the heliosphere, CR particles undergo: (i) an outward convection due the radially directed solar wind velocity, (ii) gradient, curvature and heliospheric current sheet (HCS) drift, (iii) adiabatic energy change because of the expanding solar wind, and (iv) spatial diffusion by the scattering by random magnetic irregularities; for more details, see the reviews [1-4]. The solar activity was observed at its lowest level during the minimum between solar cycles 23/24, and the Sun's activity is increased slowly after this deep and long unusual minimum, reached the maximum activity phase of solar cycle 24 in 2012-2014. This period of maximum is significantly less in terms of sunspot numbers and $10.7 \mathrm{~cm}$ solar radio flux compared to the maximum phases of previous solar cycles (https://omniweb.gsfc.nasa.gov), but the tilt angle $(\alpha)$ of the HCS still became larger than $60^{\circ}$ during this period [5], similar to what happened before (http://wso.stanford.edu/).

The Sun's magnetic field reverses its polarity during each maximum phase of solar activity. The polarity was negative ( $<0$ cycle; magnetic field lines directed outward from the Sun's southern hemisphere and inward in the northern hemisphere) during the 2001-2012 period. In April 2014, the polarity turned to positive completely ( $>0$; field lines directed outward from the Sun's northern hemisphere and inward in the southern hemisphere; see, http://wso.stanford.edu). Gradient, curvature and HCS drift models predict that positively charged CRs (e.g, protons, positrons, helium, etc.) drift inward to the inner heliosphere mainly along the equator and outward via the polar regions of the heliosphere, whereas negatively charged CRs (electrons, antiprotons, other antimatter particles) drift downward mainly from the polar regions and then outward mainly through the equatorial regions during the 2001-2012 period $(\mathrm{A}<0)$. The particles' drift direction reverses when the Sun's polarity reverses completely. This means that after 2014 electrons have begun to drift inwards along the equator while positrons drift downward from the polar regions, which will continue until the next polarity reversal period during solar maximum activity (see also [6,7]). During $\mathrm{A}<0$ cycles, positively charged CRs encounter the changing wavy HCS during their entry (as happened up to 2012), whereas electrons do so during $A>0$ cycles (as happened since the polarity reversal from $\mathrm{A}<0$ to $\mathrm{A}>0$ in 2014).

In this study, we present preliminary results, which is a continuation of the previous detailed modulation studies using a comprehensive three-dimensional (3D) heliospheric modulation model including gradient, curvature, HCS drift, applied to galactic protons [8,9], galactic electrons $[6,10]$ and galactic positrons [7,11] using PAMELA observations [12-16] during quiet heliospheric conditions (2006-2009). We consider both electrons and positrons, for a period from July 2006-May 2017, where six-month averaged PAMELA spectra over an energy range of $\sim 80 \mathrm{MeV}$ to $50 \mathrm{GeV}$ are available up to December 2009 [13-16] and Bartel rotation averaged $A M S-02$ spectra over an energy range of $\sim 1 \mathrm{GeV}$ to $50 \mathrm{GeV}$ available from May 2011-May 2017 [17]. This covers approximately one full solar cycle. Because drift patterns differ for electrons and positrons during $\mathrm{A}<0$ and $\mathrm{A}>0$ polarity phases, the availability of PAMELA positron to electron ratios from 2006 until end of 2015 [18] and similar $A M S$-02 observations [17] from 2011 until 2017 will contribute to understand how differently they get modulated during these polarity cycles, and how the major diffusion coefficients, as well as drift effects, evolve with time, from one minimum activity phase 
to the next, through a full polarity reversal period.

\section{The numerical modulation model}

A comprehensive 3D modulation model, based on the numerical solution of the Parker transport equation [TPE,19] is used to compute the differential intensity of CR electrons and positrons, over an energy range of $10 \mathrm{MeV}-70 \mathrm{GeV}$, at increasing radial distances from the Earth (1 au) up to the heliopause (122 au). The model and modulation parameters are described in detail by $[3,4,6-$ 10]. We solve the TPE for selected intervals ([7], minimum of one Bartel rotation) by using the averaged values of $\alpha$, the HMF magnitude $B$ at the Earth, and by adjusting the three diffusion and one drift coefficients.

For example, the general expression for the diffusion coefficient parallel to the average background HMF is,

$$
K_{\|}=\left(K_{\|}\right)_{0} \beta\left(\frac{B_{0}}{B}\right)\left(\frac{P}{P_{0}}\right)^{c_{1}}\left[\frac{\left(\frac{P}{P_{0}}\right)^{c_{3}}+\left(\frac{P_{k}}{P_{0}}\right)^{c_{3}}}{1+\left(\frac{P_{k}}{P_{0}}\right)^{c_{3}}}\right]^{\frac{c_{2 \|}-c_{1}}{c_{3}}},
$$

where $P$ is rigidity. It is related to the mean free path, $\lambda_{\|}=3 K_{\|} / v$, with $v$ the CR particle speed. The expression for the generalized drift coefficient is

$$
K_{D}=K_{A 0} \frac{\beta P}{3 B_{m}} \frac{\left(P / P_{A 0}\right)^{2}}{1+\left(P / P_{A 0}\right)^{2}} .
$$

The value of $K_{A 0}$ can be adjusted (between 0 to 1 ; minimum to maximum drifts) and also the scaling constant $\left(K_{\|}\right)_{0}$ to reproduce CR observations. For the two perpendicular diffusion coefficients, other parameter values in these equations, and how to adjust them in order to reproduce the PAMELA observations for the 2006-2009 minimum period are discussed in detail by [7].

\section{Very Local Interstellar Spectra for electrons and positrons}

A galactic spectrum for electrons and for positrons, more specifically a very local interstellar spectrum (LIS) has to be specified in the numerical model to be used as an initial input spectrum. which is then modulated throughout the heliosphere. This is done at the modulation boundary of the heliosphere (heliopause), specified at 122 au. Figure 1 illustrates the very LISs used for CR electrons and positrons. The electron LIS and the initially used positron LIS are computed with the GALPROP code; see examples and references in [20].

We begin with a GALPROP computed LIS for electrons to reproduce the modulated PAMELA observations from 2006-2009. Next, we use identical modulation parameters in an attempt to reproduce the PAMELA positron observations as good as the electron observations, again with a GALPROP positron LIS. But, we cannot reproduce the PAMELA positron observations satisfactory using this LIS, so we had to modify it empirically (explained in detail by [7,20]). The same GALPROP electron LIS is used then to reproduce the AMS-02 electron observations from May 


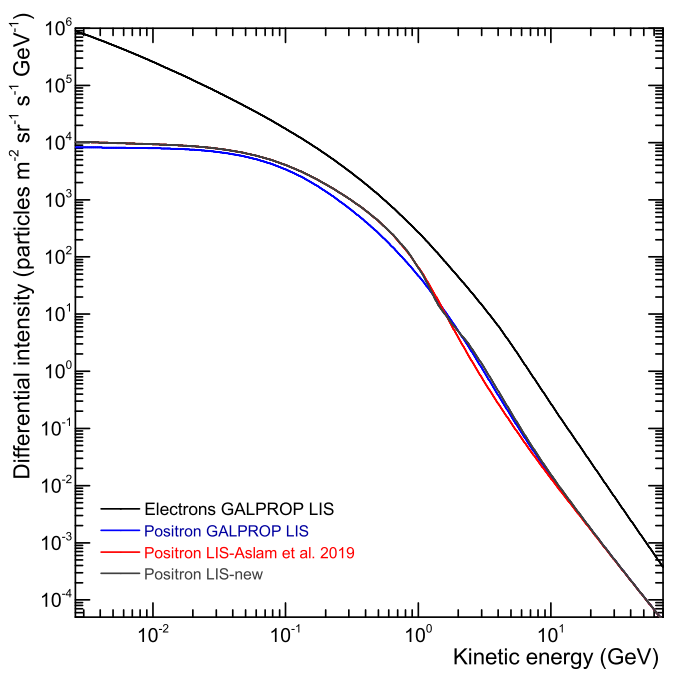

Figure 1: LIS for galactic electrons and positrons as computed with GALPROP, depict by black and blue solid lines. Red line shows the positron LIS as modified and used by [7], and the dark gray line shows the positron LIS used here but only for the period April 13-May 09, 2017 (Bartel rotation 2506).

2011-May 2017. We use the same parameters to reproduce the $A M S-02$ positron observations for the whole period, using the same modified positron LIS that reproduced the PAMELA observations from July 2006-December 2009. We can reproduce the AMS-02 positron observations for the whole period to some extent using the modified LIS but the results show some uncertainty (less satisfactory trends in reproduction) at higher energies. By modifying the LIS further at higher rigidities, we attempt to reproduce precisely the AMS-02 spectra for rotations 2501 (November 29-December 25, 2016), and 2506 (April 13-May 09, 2017). The modeling result suggests the presence of some uncertainty in the positron LIS, especially at higher rigidities (1-10 GV); see also $[7,20]$.

\section{Comparison of modeling results with observations}

We consider seven data sets of PAMELA observations, each is six-month averaged, and over an energy range of $\sim 80 \mathrm{MeV}-50 \mathrm{GeV}$, from July 2006-December 2009 and the AMS-02 observations, which is averaged over Bartel rotations, and over an energy range of $\sim 1-50 \mathrm{GeV}$, from May 2011-May 2017. From the AMS-02 observations we consider only two sets for each year, one corresponding to the middle of the year and the other one corresponding to the end of a particular year.

First, we start with the electron observations of July-December 2006, and reproduce the PAMELA spectra, shown in Figure 2(a). The averaged HMF magnitude at Earth is $4.95 \mathrm{nT}$ and the tilt angle, $\alpha=16.8^{\circ}$ during this period. In order to reproduce the electron spectrum for 2006, we have to keep $K_{A 0}=0.90$ and $\lambda_{\|}=0.438$ au at the Earth. How the HMF and $\alpha$ for each period are calculated, and the procedure of parameter selection and their values are explained in detail by [7]. Similarly, we reproduce all PAMELA spectra from 2006-2009. Figure 2(b) shows the com- 


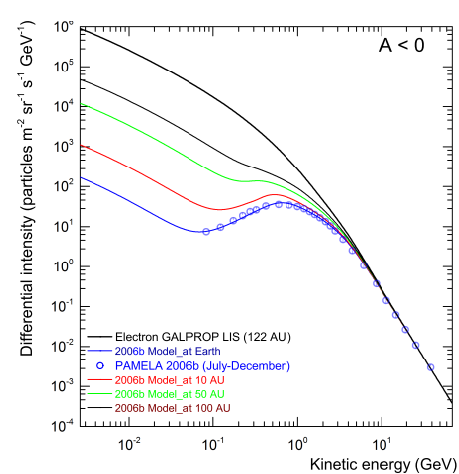

(a)

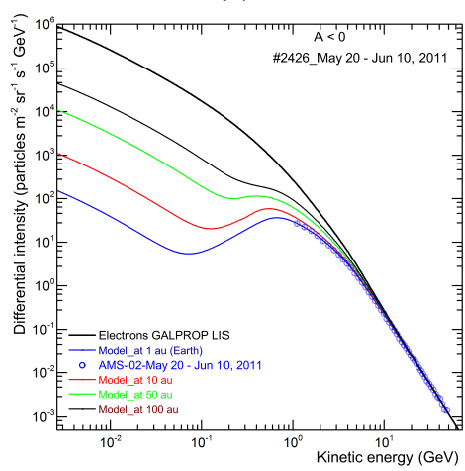

(c)

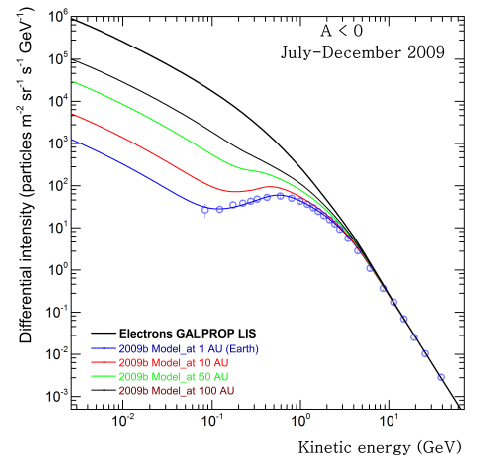

(b)

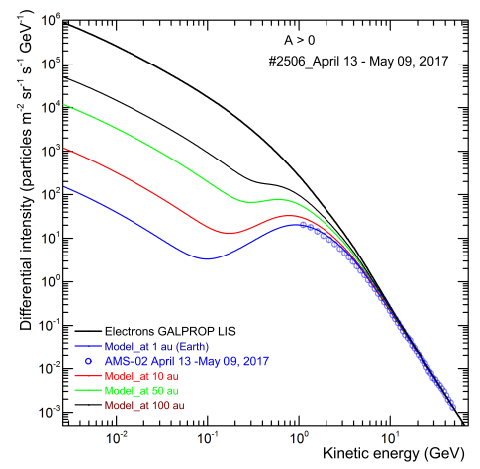

(d)

Figure 2: Computed electron spectra for (a) July-December 2006 (b) July-December 2009 (c) May 20-June 10, 2011, and (d) April 13-May 09, 2017, for increasing radial distances, at Earth (blue), $10 \mathrm{au}$ (red), $50 \mathrm{au}$ (green), and $100 \mathrm{au}$ (black). Black thick line is the GALPROP LIS specified at $122 \mathrm{au}$, the distance to the heliopause. Blue circles are PAMELA or AMS-02 electron observations for time periods as indicated.

puted spectra for July-December 2009. During this period $\alpha=9.5^{\circ}$ and $B=3.91 \mathrm{nT}$. Changing only these two values with time cannot reproduce the observed spectra, so in addition we need to change $\lambda_{\|}$to 0.593 au. Based on our understanding of electron and positron modulation, we are constrained to use the same parameter set to reproduce corresponding positron observations precisely, in which we succeed by modifying the GALPROP positron LIS. Figures 3(a) and 3(b) show the computed PAMELA positron spectra for the period July-December 2006 and July-December 2009, respectively.

Figures 2(c) and 2(d) show the simulated AMS-02 electron spectra for the periods May 20-Jun 10, 2011 (rotation 2426; start of the observations) and April 13-May 09, 2017 (rotation 2506; end of the published $A M S-02$ observations). The averaged $B=4.84 \mathrm{nT}$ and $\alpha=35.38^{\circ}$ for the 2426 rotation, with $B=5.74 \mathrm{nT}$ and $\alpha=22.49^{\circ}$ for the 2506 period. Similar to periods 2426 and 2506 , we reproduce the selected $A M S$-02 electron observations. In addition to changing $B$ and $\alpha$ values for each period, we need to adjust $K_{A 0}$ from 0 (for uncertain polarity periods) to 0.90 , and $\lambda_{\|}$from 0.289 au (solar maximum period) to 0.412 au (2011 and 2017) to reproduce these observations. Then we use the same parameter set to reproduce the positron observations, shown only starting 


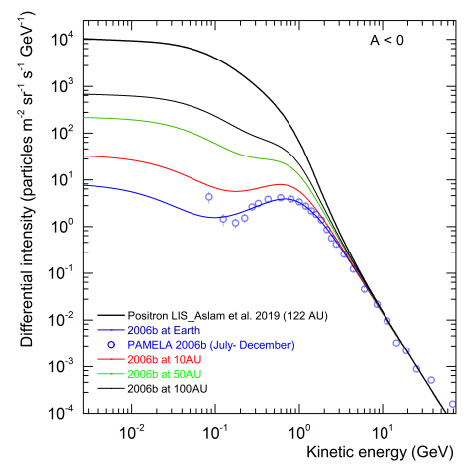

(a)

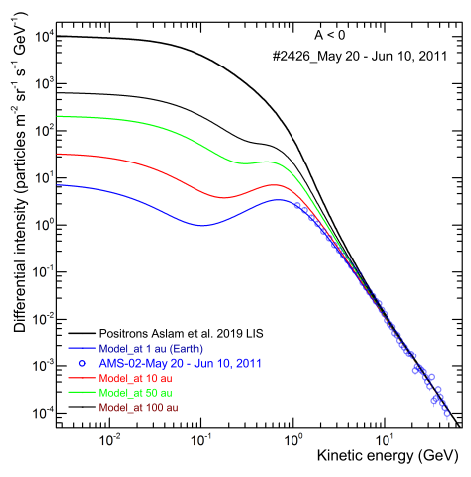

(c)

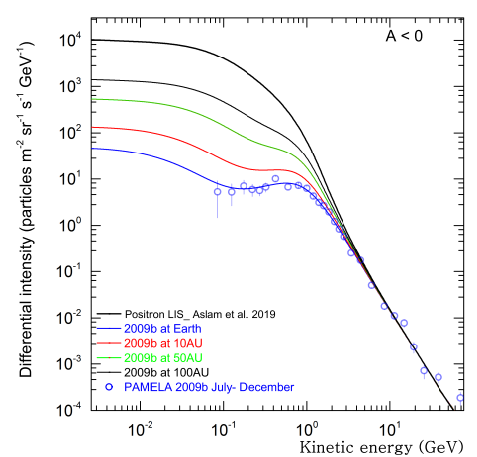

(b)

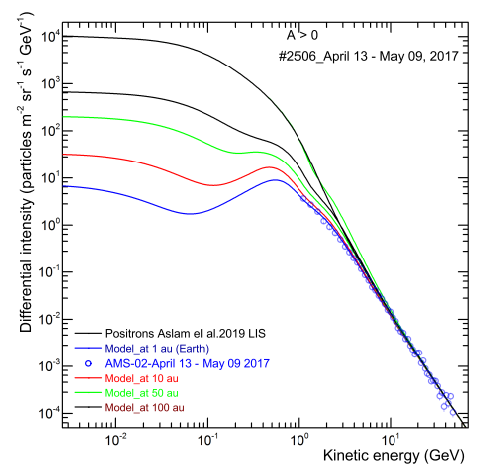

(d)

Figure 3: Computed positron spectra for (a) July-December 2006 (b) July-December 2009 (c) May 20-June 10, 2011, and (d) April 13-May 09, 2017, at different radial distances, Earth (blue), $10 \mathrm{au}$ (red), $50 \mathrm{au}$ (green), and 100 au (black). Black thick line is the modified LIS at 122 au as used by [7], except for (d), where the LIS is modified further to reproduce observations exactly). Blue circles represent PAMELA or $A M S$-02 positron observations for indicated periods.

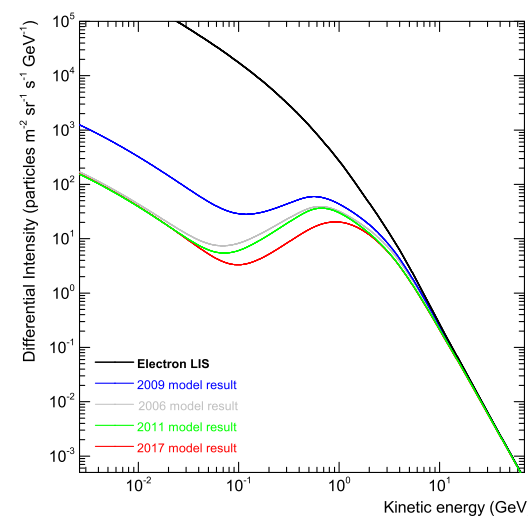

(a)

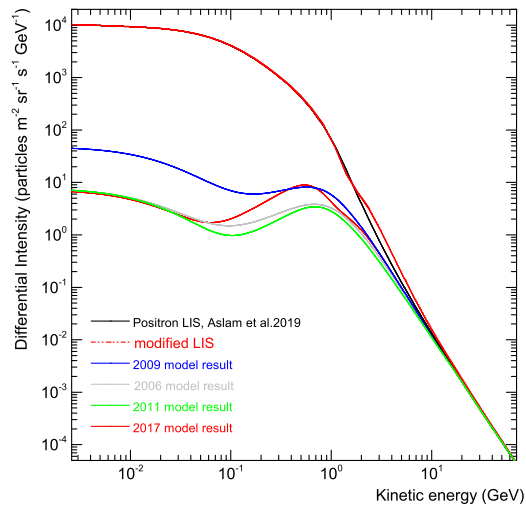

(b)

Figure 4: Computed spectra for (a) electrons and (b) positrons at Earth, for July-December 2006 (gray line), July-December 2009 (blue line), May 20-June 10, 2011 (green line) and April 13-May 09, 2017 (red line). Black solid line in (a) is the LIS; red dashed line in (b) is the new modified LIS. 
and ending data sets of AMS-02 observations in Figures 3(c) and 3(d). Some periods, especially during 2016 and 2017, show some uncertainty (less exact reproduced spectra) at higher rigidities when using the LIS that is used to reproduce the PAMELA observations. So we try a new modified LIS for April 13-May 09, 2017 to reproduce observations more exactly; see Figure 3(d). This modification of the LIS does not have any qualitative impact on our results.

Figures 4 (a) and (b) show the computed spectra of electrons and positrons at Earth (1 au) for July-December 2006, July-December 2009, May 20-June 10, 2011, and April 13-May 09, 2017. Here, the first three periods belong to the $\mathrm{A}<0$ phase but April 13-May 09, 2017 to the $A>0$ phase.

\subsection{Positron to electron ratio $\left(\mathrm{e}^{+} / \mathrm{e}^{-}\right)$}

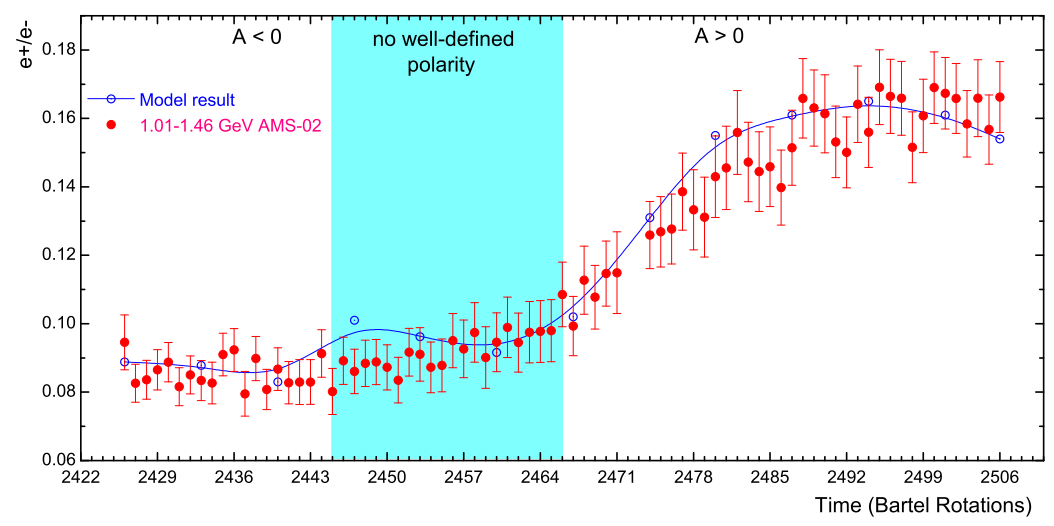

Figure 5: Computed positron to electron ratio, $\mathrm{e}^{+} / \mathrm{e}^{-}$, over the time period May 2011-May 2017 (rotations 2426-2506) for an energy range of 1.01-1.46 GeV, indicated by the solid blue line. This is compared to the $A M S-02$ observations for the same period (filled red circles with error bars). HMF reversal period during which no well-defined polarity was exhibited is indicated, with $\mathrm{A}<0$ before this reversal and $\mathrm{A}>0$ afterwards.

After reproducing both electron and positron $A M S-02$ observations, we calculate $\mathrm{e}^{+} / \mathrm{e}^{-}$, and compare our results with observations for an energy range 1.01-1.46 GeV. Figure 5 shows how $\mathrm{e}^{+} / \mathrm{e}^{-}$varies over time from 2011-2017; see also [18] who first reported this observation. The positron spectra obtained with the same LIS used by [7] for all of 2011-2017 is used for the ratio calculation (i.e. the positron spectra are reproduced here with a single LIS for the whole 20062017 period). In order to reproduce the $A M S-02$ observations we have to keep the drift coefficient at its minimum level for the solar maximum period, and then have to increase it gradually to a maximum value as solar activity decreases to a minimum. The drift coefficient is at its maximum value during the 2006-2009 minimum period, then we decrease it gradually up to its lowest value for the 2012-2014 period, to be increased gradually back to a maximum value for December 2016. Only then can we reproduce the PAMELA observed ratio [18], and as shown in Figure 5 also the $A M S$-02 observed ratio [17] precisely for this energy interval. 


\section{Summary and Conclusion}

The overall objective of this preliminary study is the reconstruction of both PAMELA and AMS-02 electron and positron spectra for the period July 2006-May 2017, also particularly the observed $\mathrm{e}^{+} / \mathrm{e}^{-}$ratio before and after the HMF polarity reversal in 2014. That is accomplished by using a 3D drift modulation model, changing the HCS tilt angle and the HMF magnitude with time, which we consider as very good proxies for solar activity. Changing their values affects the diffusion and drift coefficients directly. However, in order to reproduce and explain the observed $\mathrm{e}^{+} / \mathrm{e}^{-}$, it is necessary to adjust additionally the diffusion coefficients and very specifically the drift coefficient gradually over time, with the latter becoming zero during the HMF polarity reversal period. Only then can the observed ratio $[17,18]$ be reproduced in satisfactory detail as shown in Figure 5.

We thank the GALPROP developers for access to and use of the GALPROP WebRun service. We acknowledge the use of HCS data from the Wilcox Solar Observatory, http://wso.stanford.edu, and HMF data from NASA's OMNIWEB, http://omniweb.gsfc.nasa.gov.

\section{References}

[1] B. Heber. Space Sci. Rev. 176, 265, 2013.

[2] J. Kota. Space Sci. Rev. 176, 391, 2013.

[3] M. S. Potgieter. Living Rev. in Solar Phys. 10, 3, 2013.

[4] M. S. Potgieter. Adv. Space Res. 60, 848, 2017.

[5] O. P. M. Aslam, Badruddin. Solar Phys. 290, 2333, 2015.

[6] M. S. Potgieter, E. E. Vos, R. Munini, et al. Astrophys. J. 810, 141, 2015.

[7] O.P. M. Aslam, D. Bisschoff, M. S. Potgieter, et al. Astrophys. J. 873, 70, 2019.

[8] M. S. Potgieter, E. E. Vos, M. Boezio, et al. Solar Phys. 289, 391, 2014.

[9] E. E. Vos, M. S. Potgieter. Astrophys. J. 815, 119, 2015.

[10] M. S. Potgieter, E. E. Vos. Astron. Astrophys. 601, 23, 2017.

[11] M. S. Potgieter, E. E. Vos, D. Bisschoff, et al. Proc. Sci. (35th ICRC), 301, 44, 2017.

[12] O. Adriani, G. C. Barbarino, G. A. Bazilevskaya, et al. Astrophys. J. 765, 91, 2013.

[13] O. Adriani, G. C. Barbarino, G. A. Bazilevskaya, et al. Astrophys. J. 810, 142, 2015.

[14] R. Munini, V. di Felice, O. Adriani, et al. J. Phys.: Conference Series, 632, 012073, 2015

[15] O. Adriani, G. C. Barbarino, G. A. Bazilevskaya, et al. Phys. Rev. Lett. 111, 081102, 2013.

[16] R. Munini, V. di Felice, M. Boezio, et al. Proc. Sci. (35th ICRC), 301, 012, 2017.

[17] M. Aguilar, et al. (AMS Collaboration). Phys. Rev. Lett. 121, 051102, 2018.

[18] O. Adriani, G. C. Barbarino, G. A. Bazilevskaya, et al. Phys. Rev. Lett. 116, 241105, 2016.

[19] E. N. Parker. Astrophys. J. 128, 664, 1958.

[20] D. Bisschoff, M. S. Potgieter, O. P. M. Aslam. Astrophys. J. 878, 59, 2019. 